Unfallchirurg 2010 $113: 788$

DOI 10.1007/s00113-010-1843-3

Online publiziert: 9 . September 2010

(c) Springer-Verlag 2010

\author{
M. Schädel-Höpfner · J. Windolf \\ Klinik für Unfall- und Handchirurgie, Universitätsklinikum Düsseldorf
}

\title{
Handchirurgie in der Unfallchirurgie
}

von Handverletzungen steht der Unfallchirurg also an vorderster Front und sollte deshalb, auch bei (noch) nicht erworbener handchirurgischer Weiterbildung, im Sinne des Patienten und der eigenen Sicherheit mit allen wesentlichen anatomischen, diagnostischen und therapeutischen Aspekten der Hand vertraut sein.

\section{- Die Handchirurgie stellt ein wesentliches Tätigkeitsfeld der Unfallchirurgie dar.}

päden, Plastischen Chirurgen, aber auch insbesondere von Unfallchirurgen praktiziert. Mitunter wird hinterfragt, ob Unfallchirurgen, die sich vor allem mit polytraumatisierten Patienten und vital bedrohlichen Situationen, mit Frakturen von langen Röhrenknochen, Becken und Wirbelsäule beschäftigen, auch die komplexen und kleinen Strukturen der Hand mit dem nötigen Feingefühl und der erforderlichen Sachkenntnis operativ behandeln können. Und nicht selten wird die gegenseitige Wertschätzung der die Handchirurgie betreibenden Fächer vermisst.

Es sollten aber keine Zweifel daran bestehen, dass Handchirurgie und Unfallchirurgie untrennbar zusammengehören und Handchirurgie von Unfallchirurgen auf hohem Niveau praktiziert wird. Unfallchirurgen sind vielfach auch Handchirurgen, haben also zusätzlich zu ihrer chirurgischen Facharztweiterbildung und ihrem unfallchirurgischen Schwerpunkt die Zusatzbezeichnung Handchirurgie erworben.

Die Versorgung von Handverletzungen ist ein wesentliches Tätigkeitsfeld der Unfallchirurgie. Schließlich machen Handverletzungen trotz der erheblich verbesserten Unfallprävention immer noch etwa ein Drittel aller Verletzungen und auch aller Arbeitsunfälle aus. Bei der Vorsorgung
Leider wird diesen Tatsachen in den Weiterbildungsordnungen nur ungenügend Rechnung getragen. So machen handchirurgische Eingriffe nur 8\% im aktuellen Operationskatalog für den Facharzt für Orthopädie und Unfallchirurgie aus. Für die neue Zusatzbezeichnung Unfallchirurgie beträgt der geforderte Anteil handchirurgischer Operationen ebenfalls lediglich $8 \%$ und lag auch für die alte Schwerpunktbezeichnung Unfallchirurgie nur bei $10 \%$.

Damit wird die Verantwortung der unfallchirurgischen Kliniken, Abteilungen und Praxen deutlich, in denen Handverletzte behandelt werden, da eine Orientierung an den Vorgaben der Weiterbildungsordnungen angesichts der Häufigkeit und Relevanz von Handverletzungen zu kurz greift. Schließlich geht es um die Erhaltung oder Wiederherstellung der Handfunktion und damit der individuellen Selbständigkeit. Wichtig sind deshalb einerseits die sorgfältige und umfassende Diagnostik, die situationsgerechte Behandlung und die richtige Weichenstellung für das weitere Vorgehen. Andererseits müssen die handchirurgischen Standards der operativen Therapie vermittelt werden.
Für unfallchirurgische Weiterbilder und Weiterzubildende sollte dies gleichermaßen zu einem Prozess kontinuierlicher Wissensaneignung und praktisch-chirurgischer Vervollkommnung führen. Neuen technischen Entwicklungen, schonenden Verfahren und patientenorientierten Bewertungen sollte nach kritischer Beurteilung Gelegenheit zur Umsetzung in der klinischen Praxis gegeben werden. In diesem Sinne ist Der Unfallchirurg ein besonders geeignetes Forum für die Vermittlung neuen handchirurgischen Wissens.

Ihre

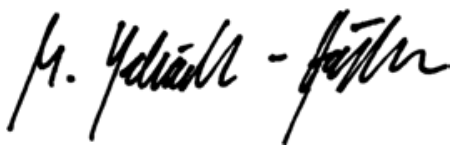

M. Schädel-Höpfner

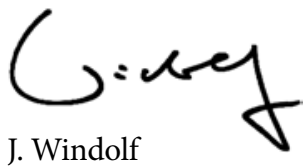

\section{Korrespondenzadressen \\ PD Dr. M. Schädel-Höpfner

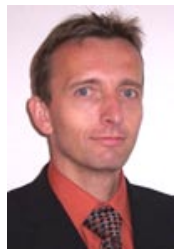 \\ Klinik für Unfall- und Handchirurgie, Universitätsklinikum Düsseldorf Moorenstraße 5, 40225 Düsseldorf schaedel@uni-duesseldorf.de}

Prof. Dr. J. Windolf

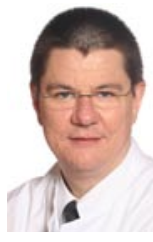

Klinik für Unfall- und Handchirurgie, Universitätsklinikum Düsseldorf Moorenstraße 5, 40225 Düsseldorf windolf@uni-duesseldorf.de 